\title{
Validity of Screening for Pre-Diabetes and Type2 DM in Patients Presenting with Oral Symptoms
}

\author{
T Muthu Vel. ${ }^{1}$, Karthikeyan S. $^{2 *}$
}

DOI: https://doi.org/10.17511/ijmrr.2021.i02.02

\footnotetext{
1 T Muthu Vel, Senior Assistant Surgeon, Government Head Quarters Hospital, Tirupur, Tamil Nadu, India.

${ }^{2 *}$ S. Karthikeyan, Assistant Professor, Department of Orthopedics, Sivagangai Medical College, Sivaganga, Tamil Nadu, India.
}

Introduction: Type $2 \mathrm{DM}$ is a major health problem in the present world with its high micro and macrovascular complications leading to increased mortality and morbidity. Our diagnosed T2DM represents the tip of the iceberg, where the hidden part places a vital challenge to the health care system. Objectives: Various risk factors are identified to screen the population for diabetes which is still an incomplete list hence we aimed to identify the dental illness as a potent risk factor to screen T2DM and Prediabetes. Methodology: This is an observational study in which 92 patients attending to dental clinic except for traumatic problems were screened for T2DM with random blood sugar and HbA1c. Results: In our study we observed $22.8 \%$ pre-diabetic and $8.8 \%$ with newly diagnosed T2DM among the study population. Chronic periodontitis was accounting for more than $60 \%$ of the subjects presenting dental illness. Conclusion: In our study we conclude that dental illness to be accounted as a potent risk factor for T2DM/Pre-Diabetes.

Keywords: Dental illness, Pre-Diabetes, T2DM, Oral symptoms

Corresponding Author

S. Karthikeyan, Assistant Professor, Department of Orthopedics, Sivagangai Medical College, Sivaganga, Tamil Nadu, India.

Email: karthidr1976@gmail.com
How to Cite this Article

To Browse

T Muthu Vel, Karthikeyan S. Validity of Screening for Pre-Diabetes and Type2 DM in Patients Presenting with Oral Symptoms. Int J Med Res Rev. 2021;9(2):66-71.

Available From

https://ijmrr.medresearch.in/index.php/ijmrr/article/ view/1268

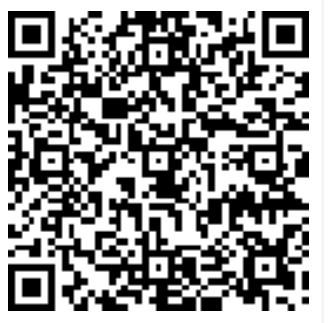

Review Round 1
2021-03-06
Funding
Nil

Review Round 3

Review Round 2

Ethical Approval Yes 


\section{Introduction}

The Indian diabetic population is predicted to reach more than 80 million by the year 2030. It indicates that immediate health policy restructuring and investment will be needed if the best use is to be made of scarce health care resources with accompanying economic constraints [1]. Currently in second place concerning the number of people with diabetes, India is expected to soon overtake China to become the 'Diabetes Capital of the World'. There is strong evidence that primary prevention of diabetes in those with prediabetes is possible. In the D-CLIP (Diabetes Community Lifestyle Improvement Program), we showed that through a step-wise program on lifestyle modification like diet, exercise and weight reduction, we could reduce diabetes incidence by 32 per cent in those with prediabetes. We have to therefore screen for prediabetes and advise lifestyle modification. As we understand the increasing burden of type 2 diabetes and its complications which includes both micro and macrovascular.

A huge financial implication also plays an important role in these aspects. As diabetes takes a toll on your entire body, but it can also increase your risk of dental disease and other symptoms that show up in your mouth. One in five cases of total loss is linked to diabetes. The oral manifestations and complications related to DM include dry mouth (xerostomia), tooth decay (including root caries), periapical lesions, gingivitis, periodontal disease, oral candidiasis, burning mouth (especially glossodynia), altered taste, geographic tongue, coated and fissured tongue, oral lichen planus (OLP), recurrent aphthous stomatitis, increased tendency to infections, and defective wound healing [2-9]. Various researches suggest that treating gum disease can help improve blood sugar control in patients having diabetes and also decreasing the progression of the disease. Practising good oral hygiene and having professional deep cleanings done by your dentist can help to reduce your HbA1c. Dental practitioners will be treating more patients with diabetes in the future, and this article provides an overview of the systemic and oral aspects of the disease that impact dental treatment [10]. With this background, we now focus more on identifying the patients in the state of pre-diabetes, where plays a major role in the hidden part of the iceberg, where the tip is the patients identified with type 2 diabetes.
Our aim of the study was to identify the incidence of prediabetes and type 2 diabetes mellitus in nondiabetic patients attending to medicine OPDs with oral illness and complaints.

\section{Methodology}

Type of study and setting: This is an observational study and intended to identify the incidence of prediabetes and type 2 diabetes mellitus in non-diabetic patients with presenting complaints of oral illness.

Place of the study: The study was carried out in the Department of Medicine, Government Headquarters hospital, Tirupur. The study was conducted in our setup since we cover both urban and rural population with a wide sector of socioeconomic status, hence we could achieve a better prediction.

Sampling method: 100 subjects were randomly selected with patients presenting with dental symptoms in the OPD willing to participate in the study.

Inclusion criteria: All patients over the age of 18 yrs presenting to the OPD with oral illness, both Male and female included. All non-diabetic patients were included in the study.

Exclusion criteria: the known case of type 2 DM, patients on steroids and OHA for other diseases, pregnancy and patients using dentures and the post-trauma case of dental problems.

Sample collection: After written consent for enrollment in the study, a detailed history was taken from all the subjects along a detailed clinical examination was done. All the subjects were investigated with random blood sugar and if found $>$ $180 \mathrm{mg} / \mathrm{dl}$ were subjected to fasting blood sugar, postprandial blood sugar. Patients were advised for HbA1c evaluation. Collected data were statistically analyzed using descriptive analysis.

\section{Results}

$\begin{aligned} & \text { Table 1: Gender distribution of study } \\
& \text { population }\end{aligned}$
\begin{tabular}{|l|l|l|}
\hline \multicolumn{1}{|c|}{ GENDER } & No. of Patients & $\%$ \\
\hline MALE & 42 & 45.65 \\
\hline FEMALE & 50 & 54.35 \\
\hline TOTAL & 92 & $100 \%$ \\
\hline
\end{tabular}


In our study we had a total of 92 patients included of which $42(45.65 \%)$ were males and $50(54.35 \%)$ were females.

Table 2: Age distribution of study population

\begin{tabular}{|l|l|l|l|l|}
\hline \multicolumn{1}{|c|}{ AGE (IN YEARS) } & MALE & FEMALE & Total & \multicolumn{1}{c|}{$(\%)$} \\
\hline $20-30$ & 4 & 6 & 10 & 10.9 \\
\hline $31-40$ & 14 & 7 & 21 & 22.8 \\
\hline $41-50$ & 11 & 21 & 32 & 34.78 \\
\hline $51-60$ & 8 & 8 & 16 & 17.39 \\
\hline$>60$ & 5 & 8 & 13 & 14.13 \\
\hline Total & 42 & 50 & 92 & 100 \\
\hline
\end{tabular}

In our study of 92 patients we had an age distribution ranging from 22 lowest to 78 years eldest. In our study we had more than $50 \%$ between 31 to 50 years which gives an impression more common among middle-age who is an active earning member of the family.

Table 3: HbA1c level in the study population

\begin{tabular}{|l|l|l|}
\hline \multicolumn{1}{|c|}{ HbA1c } & \multicolumn{1}{|c|}{ No. of Patients } & \multicolumn{1}{c|}{$\%$} \\
\hline$<5.7$ & 62 & 68.5 \\
\hline $5.7-6.5$ & 22 & 22.8 \\
\hline$>6.5$ & 8 & 8.7 \\
\hline
\end{tabular}

In our presentation we intended to do all subjects with HbA1c as it should be more specific for the diagnosis of Type 2 diabetes. We had a wide range of $\mathrm{HbA} 1 \mathrm{c}$ from 4.7 to $9.8 \%$ in our study population. There were 8 patients $(8.7 \%)$ with HbA1c of more than $6.5 \%$ whom we confirm Type 2 DM, 22 patients $(22.8 \%)$ were in the range of 5.7 to $6.5 \%$ whom it implies as pre-diabetes which is the important group whom we try to identify at the earliest. In our study 62 patients $(68.5 \%)$ with less than $5.7 \% \mathrm{HbA} 1 \mathrm{c}$ in the non-diabetic range.

Table 4: Dental problems

\begin{tabular}{|l|l|l|}
\hline \multicolumn{1}{|c|}{ Dental problems } & \multicolumn{1}{c|}{ No. of patients } & \multicolumn{1}{c|}{$\%$} \\
\hline Dental carries & 14 & 15.21 \\
\hline Chronic Gingivitis & 23 & 25 \\
\hline Chronic periodontitis & 59 & 64.13 \\
\hline Dentoalveolar abscess & 01 & 1.08 \\
\hline Periodontal abscess & 03 & 3.26 \\
\hline Gum hypertrophy & 02 & 2.17 \\
\hline Total & 92 & 100 \\
\hline
\end{tabular}

In our study where we identified people with a nontraumatic oral disease were identified to evaluate for the presence of Type 2DM and pre-diabetes. Among the Dental illness profile -Dental caries 14(15.21\%), Chronic Gingivitis 23(25\%), Chronic Periodontitis 59(64.13\%), Dentoalveolar abscess
$1(1.08 \%)$, Periodontal $3(3.26 \%)$ and Gum Hypertrophy 2 (2.17\%). Chronic Periodontitis was the most common manifestations with more than $60 \%$ of patients.

\section{Discussion}

Diabetes mellitus (DM) is a chronic metabolic disease characterized by hyperglycaemia due to either a deficiency of insulin secretion or resistance to the action of insulin or both [2-4]. Chronic hyperglycemia can lead to different complications in various regions of the body including the oral cavity, so blood glucose control is very critical [5]. Possible mechanisms that may be related to oral complications of diabetes include impaired neutrophil function, increased collagenase activity, and a reduction in collagen synthesis, microangiopathy, and neuropathy [5]. Oral complications in patients with DM are considered major complications of the disease and can impress the patients' quality of life. There is evidence that chronic and persistent oral complications in these patients adversely affect blood glucose control.

Type 2 DM is a disease that affects all parts of the body and each organ. In the same way it is well understood that it's a risk factor for dental illness (Periodontal disease) but the reverse might be true as various research proves the same but it's less established in the developing countries as dental problems are commonly neglected. A meta-analysis of four studies with a total of 3,524 adults ( $>18$ years old) showed those with diabetes have a twofold higher risk of developing the periodontal disease compared to those without diabetes [2]. Desvarieux and Demmer [1] both noted that the incidence of diabetes odds was increased by $40 \%$ among participants with gingivitis $(P<0.05)$ and by $50 \%$ among participants with periodontitis $(P<0.05)$ compared with periodontal healthy participants. Periodontal diseases and diabetes mellitus are closely associated and are highly prevalent chronic conditions. Possible mechanisms for an explanation of increased susceptibility to periodontal diseases include alterations in host defence response (such as neutrophil dysfunction), subgingival microflora, structure and metabolism of collagen, vascularity, and gingival crevicular fluid and also, inheritance patterns. Furthermore, several risk factors have been reported, which make these patients more susceptible to the development of periodontal disease including poor oral hygiene, poor metabolic control, longer duration of diabetes, and smoking. 
Inflammation is a critical player in the association, and its importance is just now coming to light. Diabetes increases the risk of periodontal diseases as demonstrated by several plausible mechanisms [3]. P. M. Preshaw \& A. L. Alba et al [4] in their study confirm that diabetes is a significant risk factor for periodontitis, and the risk of periodontitis is greater if glycaemic control is poor; people with poorly controlled diabetes.

With the above evidence we aimed to study the incidence of prediabetes and type 2 diabetes mellitus in non-diabetic patients attending a dental clinic in a rural population. Our study showed predominant age group attending the dental clinic were between 41 to 50 years. We found more than $50 \%$ with periodontal disease. The incidence of prediabetic was $22.8 \%$ and the incidence of T2DM was $8.7 \%$.

Interestingly, and as-synthesized in a 2018 systematic review [5], a significant proportion of dental patients denying having diabetes, namely almost one-third of dental patients both in Denmark [6], Saudi Arabia [7], and the US [8-10] had prediabetes or manifest diabetes; or were considered having high risk therefore [11].In Great Britain, $29.5 \%$ were considered moderate-risk and $18.1 \%$ high risk for diabetes, for a total of $47.6 \%$ [12].

A US study in Manhattan reported $8.6 \%$ potentially having diabetes and $46.5 \%$ having pre-diabetes, for a total of 54.1 [13]; and in Washington State, $45 \%$ of adults had $\mathrm{HbA} 1 \mathrm{C}>5.6 \%$ [14]. Among 2003 Nigerians, $4.4 \%$ had random plasma glucose levels of $>200 \mathrm{mg} / \mathrm{dl}$ [15]. Notably, there was a doseresponse pattern of increasing levels of hyperglycemia with increasing severity of periodontitis [16]. With the above analysis we paid attention to the important role of oral health as a novel tool for medical care professionals in the prevention and management of diabetes of any type.

\section{Conclusion}

In our study we conclude that dental illness to be accounted as a potent risk factor for T2DM/PreDiabetes. Screening for DM in dental practice and awareness of oral health applied in medical practice, both followed up by proper reciprocal referrals and potential co-management of mutual patients by medical and dental professionals should have good potential for benefiting the healthcare.
As we clearly understand from the literature that oral health and Diabetes Mellitus are intractable it's clear to have a dental clinic in all diabetology clinics and screening of diabetes is mandated in Dental clinics.

\section{Contribution by authors}

T MuthuVel had conceptualized the study, prepared the study protocol, conducted the data collection, analysis and manuscript writing. T MuthuVel has verified all the drafts and approved the final draft., S. Karthikeyan had provided key inputs on methodology during protocol preparation, supported data compilation and analysis.

\section{Reference}

01. Balasheb Bansode, Dr Suresh Jungari et al. Economic burden of diabetic patients in India- A review. Diabetes Metab Synd. Jul-Aug 2019;13(4)2469-2472. doi: $10.1016 /$ j.dsx.2019.06.020 [Crossref]

02. Cicmil S, Mladenović I, Krunić J, Ivanović D, Stojanović N. Oral Alterations in Diabetes Mellitus. Balk J Dent Med. 2018;22;7-14. doi: $10.2478 / \mathrm{bjdm}-2018-0002$ [Crossref]

03. Al-Maskari AY, Al-Maskari MY, Al-Sudairy S. Oral Manifestations and Complications of Diabetes Mellitus- A review. Sultan Qaboos Univ Med J. 2011;11;179-186.

[Crossref]

04. Indurkar MS, Maurya AS, Indurkar S. Oral Manifestations of Diabetes. Clin Diabetes. 2016;34;54-57.

DOI: $10.2337 /$ diaclin.34.1.54 [Crossref]

05. Cicmil A, Govedarica O, Lečić J, Mališ S, Cicmil $S$, Čakić S. Oral Symptoms and Mucosal Lesions in Patients with Diabetes Mellitus Type 2. Balk J Dent Med. 2017;21;50-54.

DOI: $10.1515 /$ bjdm-2017-0007 [Crossref]

06. Mauri-Obradors E, Estrugo-Devesa A, JanéSalas $E$, Viñas $M$, López-López J. Oral manifestations of Diabetes Mellitus- $A$ systematic review. Med Oral Patol Oral Cir Bucal. 2017;22;e586-e594.

DOI: $10.4317 /$ medoral.21655 [Crossref] 
07. Trentin MS, Verardi G, De C Ferreira M, de Carli JP, da Silva SO, Lima IF, Paranhos LR. Most Frequent Oral Lesions in Patients with Type 2 Diabetes Mellitus. J Contemp Dent Pract. 2017;18; 107-111.

[Crossref]

08. Ship JA. Diabetes and oral health- an overview. J Am Dent Assoc. 2003;134 Spec No- 4S-10S. [Crossref]

09. Khan T. Oral manifestations and complications of diabetes mellitus- A review. Int J Med Health Res. $2018 ; 4 ; 50-52$.

DOI: $10.22271 /$ ijmhr [Crossref]

10. Jonathan A ship, et al. Diabetes and oral healthan overview. J Am dental asso. 2003 Oct;134 Spec No- 4S-10S.

Doi: 10.14219/jada.archive.2003.0367 [Crossref]

11. Demmer RT, Jacobs DR, Desvarieux $M$. Periodontal disease and incident type 2 diabetes. Diabetes Care. 2008;31;1373-1379. [Crossref]

12. Papapanou PN. Periodontal diseasesepidemiology. Annals of Periodontology. $1996 ; 1(1) 1-36$.

[Crossref]

13. Renata S Leite, Nicole M Marlow, Jyotika K Fernandes, et al. Oral health and type 2 diabetes. Am J Med Sci. 2013 April;345(4)271273.

doi: 10.1097/MAJ.0b013e31828bdedf [Crossref]

14. PM Preshaw, A L Alba, D Herrera, S Jepsen et al. Periodontitis and diabetes- a two-way relationship. Diabetologia. 2012;55;21-31.

DOI $10.1007 /$ s00125-011-2342 [Crossref]

15. Glurich I, Bartkowiak B, Berg RL, Acharya A. Screening for dysglycaemia in dental primary care practice settings- systematic review of the evidence. Int Dent J. 2018;68;369-77. doi: $10.1111 /$ idj.12405 [Crossref]

16. HolmNCR, BelstrømD, ØstergaardJA, SchouS, HolmstrupP, Grauballe MCB. Identification of individuals with undiagnosed diabetes and prediabetes in a Danish cohort attending dental treatment. J Periodontol. 2016;87;395-402.

doi: 10.1902/jop.2016.150266 [Crossref]
17. AlGhamdi AS, Merdad K, Sonbul H, Bukhari SM, Elias WY. Dental clinics as potent sources for screening undiagnosed diabetes and prediabetes. Am J Med Sci. 2013;345;331-4.

doi: 10.1097/MAJ.0b013e318287c96c [Crossref]

18. Genco RJ, Schifferle RE, Dunford RG, Falkner $\mathrm{KL}$, Hsu WC, Balukjian J. Screening for diabetes mellitus in dental practices- a field trial. J Am Dent Assoc. 2014;145;57-64.

doi: $10.14219 /$ jada.2013.7 [Crossref]

19. Herman WH, Taylor GW, Jacobson JJ, Burke R, Brown MB. Screening for prediabetes and type 2 diabetes in dental offices. J Public Health Dent. $2015 ; 75 ; 175-82$.

doi: $10.1111 /$ jphd.12082 [Crossref]

20. Kalladka M, Greenberg BL, Padmashree SM, Venkateshaiah NT, Yalsangi S, Raghunandan BN, et al. Screening for coronary heart disease and diabetes risk in a dental setting. Int J Public Health. $2014 ; 59 ; 485-92$.

doi: 10.1007/s00038-013-0530-x [Crossref]

21. Estrich CG, Araujo MWB, Lipman RD. Prediabetes and diabetes screening in dental care settings- NHANES 2013 to 2016. JDR Clin Trans Res. 2019;4;76-85.

Doi: $10.1177 / 2380084418798818 \quad$ [Crossref]

22. Wright D, Muirhead V, Weston-Price S, Fortune F. Type 2 diabetes risk screening in dental practice settings- a pilot study. $\mathrm{Br}$ Dent $\mathrm{J}$. 2014;216;E15.

doi: $10.1038 /$ sj.bdj.2014.250 [Crossref]

23. Lalla E, Cheng B, Kunzel C, Burkett S, Lamster IB. Dental findings and identification of undiagnosed hyperglycemia. J Dent Res. 2013;92;888-92.

doi: $10.1177 / 0022034513502791 \quad$ [Crossref]

24. Franck SD, Stolberg RL, Bilich LA, Payne LE. Point-of-care HbA1c screening predicts diabetic status of dental patients. J Dent Hyg. $2014 ; 88 ; 42-52$.

[Crossref] 
25. Opeodu OI, Adeyemi BF. Undiagnosed diabetes mellitus- a survey of dental outpatients in a tertiary hospital. Afr] Med Med Sci. $2013 ; 42 ; 39-45$.

[Crossref]
26. Harase T, Nishida W, HamakawaT, Hino S, Shigematsu K, Kobayashi $S$, et al. Clinical implication of blood glucose monitoring in general dental offices- the Ehime Dental Diabetes study. BMJ Open Diab Res Care. $2015 ; 3$.

doi: $\quad 10.1136 /$ bmjdrc-2015-000151 [Crossref] 